\title{
Multicolour Emission States from Charge Transfer between Carbon Dots and Surface Molecules
}

\author{
Shengliang Hu ${ }^{1, *}$, Yanbing Wang ${ }^{1}$, Wenyu Zhang ${ }^{1}$, Qing Chang ${ }^{1, *}$ and Jinlong Yang ${ }^{1,2}$ \\ 1 School of Material Science and Engineering, North University of China, Taiyuan 030051, China; \\ wybingzbdx@yeah.net (Y.W.); zwyu2017@163.com (W.Z.), jinlongnuc@yeah.net (J.Y.) \\ 2 State Key Laboratory of New Ceramics and Fine Processing, Tsinghua University, Beijing 100084, China \\ * Correspondence: hsliang@yeah.net (S.H.); changneu@gmail.com (Q.C.); Tel.: +86-351-3559638 (S.H.)
}

Academic Editor: Lioz Etgar

Received: 13 December 2016; Accepted: 26 January 2017; Published: 11 February 2017

\begin{abstract}
The emissive states of carbon dots have been tuned by controlling the charge transfer process. The carbon dots couple with molecules, which are made of a benzene ring and different heteroatom substituents, through amino-carboxylic bonds that are generally identified as charge transfer promoters at the interface. New ways of radiative recombination are created due to the transfer of photo-excited electrons from carbon dots to the lowest unoccupied molecular orbital (LUMO) of the grafted molecules. By variation of the molecular orbital energy levels via heteroatom substituents in the benzene ring, the different optical properties and emission colors of the carbon dots were presented. This work opens up new opportunities for the application of carbon dots since different heteroatom substituents could lead to many possibilities for conjugation with drugs and biomolecules.
\end{abstract}

Keywords: carbon dots; band gap; surface states; charge transfer; photoluminescence; heteroatom groups

\section{Introduction}

Carbon dots (CDs), a class of zero-dimensional carbongenic nanomaterials with bright fluorescence, have attracted considerable attention because of their elemental nontoxicity, facile synthesis, and their potential applications in imaging, sensing, lighting, catalysis, and photovoltaics [1-5]. The photoluminescence (PL) from CDs are highly sensitive to a number of factors [6]. For the CDs resembling the crystalline structure of single or a-few layered graphene (called graphene quantum dots, GQDs), the theoretical modelling and calculations suggest that their energy gaps can be tuned by the lateral size, shape, edge configuration, and thickness [7-10]. However, completely consistent results are difficult to be observed in experiments [6]. This could be caused by the huge heterogeneity of individual particles from the preparation sample. In addition, the theoretical calculations are generally limited without taking all factors into account simultaneously $[8,10]$.

For quasi-spherical CDs, many works have demonstrated that their PL tuning depends more on surface states instead of $\mathrm{sp}^{2}$ clusters in the core, though the quantum confinement effect also plays a role $[4,6,11,12]$. Such CDs have ultrahigh specific surface areas that enables their surface-facet atoms to be as important as those on the inside. Therefore, surface incorporation methodologies such as heteroatom incorporation and surface functionalization have become effective ways to tune the electronic and opto-electronic properties of CDs [11,13-17]. Recent studies have demonstrated the key roles that $\mathrm{O}$ and $\mathrm{N}$ elements or their related groups play in engineering band gaps of CDs when they are incorporated into their external lattice framework [17-24]. Other elements such as $S$ and $P$ could enhance the effects of $\mathrm{O}$ and $\mathrm{N}$-contained groups on the band gaps in $\mathrm{CDs}$ through a cooperative effect [25-30]. Multi-color light-emitting CDs were obtained by tuning $\mathrm{O}$ and/or $\mathrm{N}$ concentrations and their bonding types with $C$ in CDs [12,18-20,22-24,31-34]. In principle, it is generally accepted that 
the incorporation of heteroatoms in the carbon lattice disrupts $\mathrm{sp}^{2}$ hybridization of carbon atoms and thereby results in the formation of new band gaps in CDs [12,18,20,22,34].

Besides the above mechanism of tailoring the band structure by heteroatom-induced defect states and energy traps, charge transfer, which is a basic process governing the electronic and opto-electronic properties of a material, can be further used to tune emissive sites in CDs $[15,35,36]$. Sun's group has firstly demonstrated the ability of CDs to transfer their surface-confined electrons and holes [37]. Then Jin et al. provided the experimental and theoretical evidence in support of charge-transfer-controlled electron-hole recombination [35]. They found the electron transfer from amine groups to six-member carbon rings, causing the variation of band gaps in CDs. However, the change of band gaps is still limited for CDs by attaching amine groups. Accordingly, developing a molecule implantation scenario for specially tuning emissive sites in CDs through the charge transfer effect is essential not only for the application of CDs but also for understanding their interaction with surface-attached molecules.

Here we present a new approach to tuning emissive states in CDs by grafting molecules consisting of a benzene ring and different heteroatom groups on the surface of CDs through amino-carboxylic bonds. In this system, the amino-carboxylic molecular junction acts as a conduit for electron transfer between the CDs and the surface-attached molecules, while the heteroatom groups bonding with the benzene ring are employed to regulate the molecular orbital energy levels.

\section{Experimental Section}

CDs with abundant $-\mathrm{COOH}$ groups, named as $\mathrm{CDs}-\mathrm{COOH}$, were firstly fabricated by solvothermally treating citric acids [38]. Subsequently they were modified with p-phenylenediamine (S1), sulfanilic acid (S2), and 4-aminothiophenol (S3), respectively, through acylation reaction forming amino-carboxylic bonds (Figure 1).

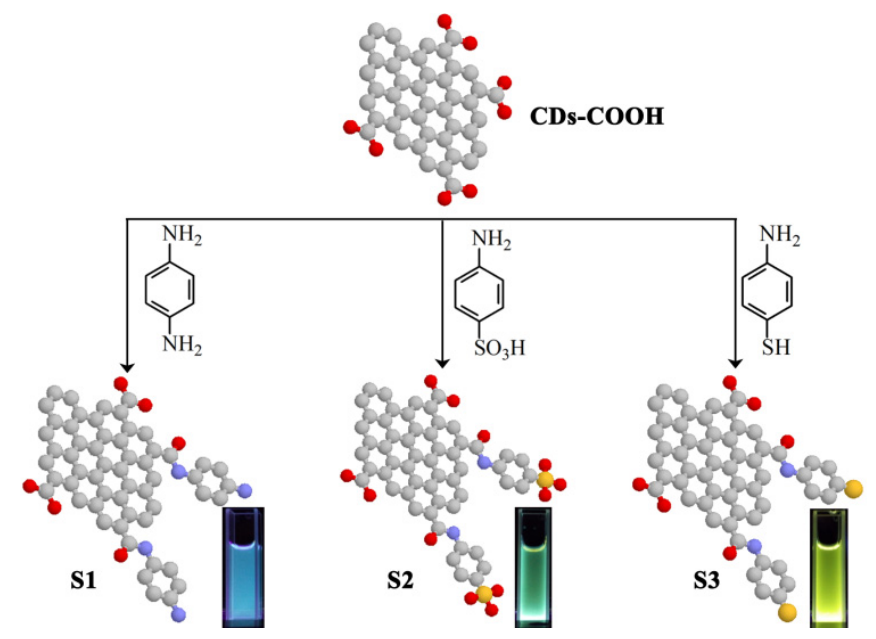

Figure 1. Schematic of modifying CDs with p-phenylenediamine (S1), sulfanilic acid (S2), and 4-aminothiophenol (S3), respectively, and their corresponding emission photos under UV excitation of $356 \mathrm{~nm}$.

Preparation of CDs-COOH followed procedures reported previously [38]. Briefly, $1.68 \mathrm{~g}$ of citric acid was dissolved in $40 \mathrm{~mL}$ of DI water. The obtained solution was transferred to a Teflon-lined autoclave $(100 \mathrm{~mL})$ and then heated at $200{ }^{\circ} \mathrm{C}$ for $5 \mathrm{~h}$. After cooling to room temperature naturally, the transparent solution containing $\mathrm{CDs}-\mathrm{COOH}$ was obtained by $30 \mathrm{~min}$ centrifugation $(10,000 \mathrm{rpm})$. $0.25 \mathrm{mmol}$ of p-phenylenediamine, sulfanilic acid, or 4-aminothiophenol were dissolved into the same volume of $\mathrm{CDs}-\mathrm{COOH}$ solution $(10 \mathrm{~mL})$, respectively. Then the solution was put into a $50 \mathrm{~mL}$ Teflon-lined autoclave and was heated at $135^{\circ} \mathrm{C}$ for $5 \mathrm{~h}$ again. Thus, the molecule-modified CDs, i.e., S1, 
S2, and S3, respectively, were obtained after cooling to room temperature. To compare, the samples were also prepared by changing the amounts (e.g., $0.125,0.375 \mathrm{mmol}$ ) of the p-phenylenediamine, sulfanilic acid, or 4-aminothiophenol under the same conditions. Moreover, the samples were also prepared by the same process with reaction temperature variation (e.g., 70, 90, 160, $200{ }^{\circ} \mathrm{C}$ ). All sample solutions were adjusted to the same $\mathrm{pH}$ value by neutralization with base.

All photoluminescence (PL) spectra were obtained by a fluorescence spectrophotometer (Hitachi, Tokyo, Japan). X-ray photoelectron spectroscopy (XPS) data of all samples was collected by a Kratos AXIS 165 multitechnique electron spectrometer (Shimadzu, Tokyo, Japan) with an $\mathrm{Al} \mathrm{K} \alpha \mathrm{X}$-ray source for determining the composition and chemical bonding configurations. Transmission electron microscopy (TEM) measurements were performed on a Tecnai G2 F20 electronic microscope (FEI, Fremont, CA, USA). UV-Vis absorption was characterized by a UV-2550 UV-vis spectrophotometer (Shimadzu, Tokyo, Japan). The time-resolved PL spectra were obtained on an FLS 920 PL spectrometer (Edinburgh Instruments, Livingston, UK). The infrared spectra were collected on a Thermo Nicolet 360 FT-IR spectrophotometer (Thermo Fisher Scientific, Waltham, MA, USA).

\section{Results and Discussion}

As shown in Figure 1, three kinds of heteroatom groups, i.e., $-\mathrm{NH}_{2},-\mathrm{SO}_{3} \mathrm{H}$, and $-\mathrm{SH}$, are also introduced in CDs with molecule implantations. Under the $365 \mathrm{~nm}$ UV lamp, the markedly different emission colors were observed from the obtained samples of S1, S2 and S3, respectively.

Figure 2 shows the PL spectra of CDs-COOH, S1, S2, and S3 excited with the different light wavelengths. For each sample of CDs, there were one strongest PL peak and one optimal excitation wavelength, reflecting the most dominant emissive sites in the CDs. The strongest emission peaks of CDs-COOH, S1, S2, and S3 are around 425, 465, 500, and $530 \mathrm{~nm}$, respectively. A striking shift in PL emission with varying excitation is observed in $\mathrm{CDs}-\mathrm{COOH}$ and $\mathrm{S} 1$ (Figure 2a,b). However, there is an obvious difference in the PL spectra other than the PL peak variations. The redshift degree for $\mathrm{CDs}-\mathrm{COOH}$ is proportional to the increase of the excitation wavelength, whilst the PL peaks of S1 do not change obviously when the excitation wavelength is smaller than the optimal wavelength. Both S2 and S3 reveal excitation-wavelength-independent PL properties, but their optimal excitation wavelengths are still different (Figure 2c,d). In order to offer insight into these differences of PL behaviors presented in Figure 2, we performed further characterizations of sizes and compositions, which are possible factors for tuning the band gaps in CDs [12].
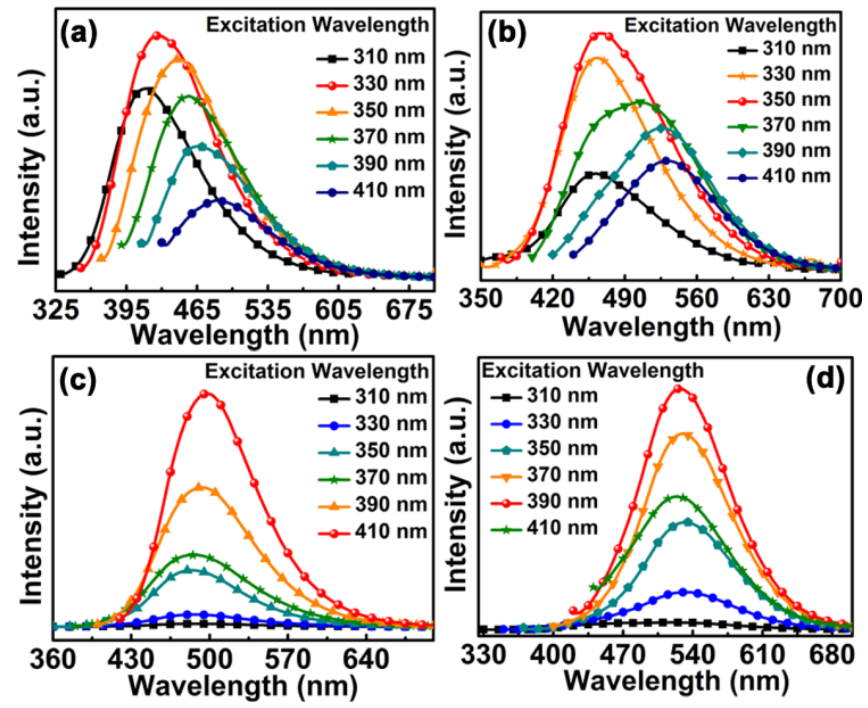

Figure 2. PL spectra of (a) CDs-COOH, (b) S1, (c) S2, and (d) S3 excited with different wavelengths. 
The TEM images reveal that CDs from the samples of CDs-COOH, S1, S2, and S3 are well dispersed (Figure 3a) and exhibit similar size distribution (Figure 3b). Moreover, the lattice fringes of CDs show the same interplanar spacing on the basis of their high resolution TEM (HRTEM) images (Figure S1). Although the concentration of CDs could also modulate their PL centers according to the reports of Yu's group [39], this case was not observed in this work. Accordingly, the differences in PL from CDs-COOH, S1, S2, and S3 could be independent of their sizes.
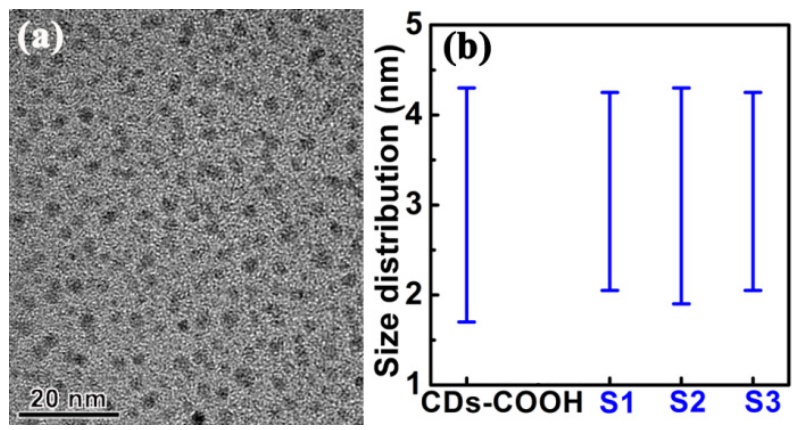

Figure 3. A typical TEM image of (a) CDs and (b) the size distributions of CDs-COOH, S1, S2, and S3.

The surface compositions of CDs were determined by XPS, which has become a main tool that is widely used to characterize these materials. A similar $\mathrm{C} 1$ s signal is observed from the $\mathrm{CDs}-\mathrm{COOH}, \mathrm{S} 1$, S2, and S3 samples, as shown in Figure 4a. There are clearly three different chemical environments, corresponding to $\mathrm{C}-\mathrm{C}, \mathrm{C}-\mathrm{X}(\mathrm{X}=\mathrm{O}, \mathrm{N}$, or $\mathrm{S})$, and $\mathrm{N}-\mathrm{C}=\mathrm{O} / \mathrm{O}-\mathrm{C}=\mathrm{O}$ bonds, respectively $[12,20,23,26,28]$. The N element is presented in all the CDs from S1, S2, and S3 due to molecular modification (Figure $4 \mathrm{~b}$ ). Their N1s XPS spectra are nearly identical and can be resolved into two peaks at 399.7 and $401.2 \mathrm{eV}$, which are attributed to the $\mathrm{C}-\mathrm{N}-\mathrm{C}$ (pyrrolic $\mathrm{N}$ ) and $\mathrm{N}-\mathrm{H}$ bonds, respectively $[28,29,31]$. This also implies the presence of amino-carboxylic bonds in S1, S2, and S3 after molecule modification. Besides the signals of $C, N$, and $O$, the S2p peaks at different binding energies are observed in CDs from S2 and S3, respectively (Figure 4c,d). According to the high resolution scan of S2p, the bonds of C-S and $\mathrm{S}=\mathrm{O}$ are present in the $\mathrm{CDs}$ from $\mathrm{S} 2$, while the $\mathrm{S} 2 \mathrm{p}_{3 / 2}$ and $\mathrm{S} 2 \mathrm{p}_{1 / 2}$ of $\mathrm{C}-\mathrm{S}-\mathrm{H}$ bonding exist in those from S3 [25-28], suggesting that there are different S-contained groups in the CDs from S2 and S3. Infrared (IR) spectra (Figure S2) confirmed the presence of vibrational absorption bands of $\mathrm{C}=\mathrm{O}$ from $\mathrm{CONH}$, and $\mathrm{C}-\mathrm{N}$ in $\mathrm{CDs}$ modified by molecules, and revealed the stretching peaks of the $-\mathrm{SO}_{3} \mathrm{H}$ and $-\mathrm{SH}$ groups in the CDs from S2 and S3, respectively. The above results clearly demonstrate that the molecules of p-phenylenediamine, sulfanilic acid, and 4-aminothiophenol are grafted on CDs through amino-carboxylic bonds and then give rise to the changes of their chemical structures and compositions. This could result in the PL tuning of CDs. 


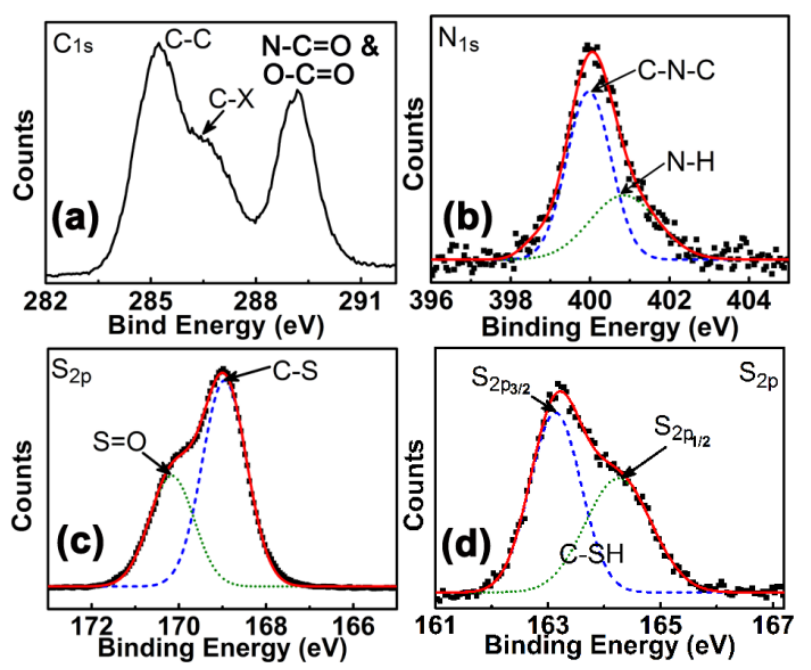

Figure 4. XPS spectra for modified CDs (a) a typical C1s; (b) N1s; (c) S2p of S2 and (d) S2p of S3.

Previous works have postulated that the low PL efficiency of only $O$-incorporated CDs is not only highly associated with many kinds of possible recombination, but is attributed to the charge transfer induced by $\mathrm{C}=\mathrm{O}$ bonds $[40,41]$. Consequently, both heteroatom incorporation and molecule implantation are always used for suppressing nonradiative recombination and charge transfer to improve the PL of CDs $[2,13,25,31]$. For instance, $\mathrm{Li}$ et al. demonstrated that the absorption and PL of CDs were modified after they were conjugating with polyethylene glycol (PEG) polymers of different molecular weights via amino-carboxylic bonds [42]. According to the work reported by Kladnik et al., however, the amino-carboxylic bonds connecting CDs with the surface molecules can serve as a fast tunnel of charge transfer [43]. Why do the CDs after molecular modification exhibit brighter luminescence than the $\mathrm{CDs}-\mathrm{COOH}$ in the present work? This can be ascribed to the benzene ring in the molecule structure employed, which is an antibonding state and responsible for reserving electrons or energy [35]. Thus, the possibility that the excitation energy returns to the ground state by emitting a photon rather than being lost as heat is enhanced.

It has been confirmed that a series of electronically excited states can be created in CDs with $\mathrm{O}$ incorporation $[20,23]$. When the energy levels of these excited states are higher than those of the molecular orbital of the molecules grafted on the surface of the CDs, the electrons excited in CDs will be quickly transferred to their lowest unoccupied molecular orbital (LUMO) through the amino-carboxylic molecular junction as shown in Figure 5a. Because the electron density in the benzene ring highly depends on its substituent groups [35], their changes will significantly influence the LUMO levels of superficial molecules and then cause band gap shifts of molecule-modified CDs. For instance, the electron density in the benzene ring can be reduced by the $\mathrm{SO}_{3} \mathrm{H}$ groups as an electron-withdrawing substituent; whereas it can be enhanced by the $\mathrm{SH}$ groups possessing strong electron-donating abilities. From Figure 5b, a typical new absorption band at around $250 \mathrm{~nm}$, which is attributed to the benzene ring, is presented after the molecular modifications of $\mathrm{CDs}-\mathrm{COOH}$. Remarkably, a redshift of this absorption band can be observed by comparing S2 with S3, due to the increased electron density in benzene ring. To further verify this, $\mathrm{S} 3$ was treated with $\mathrm{HNO}_{3}$, thus removing the $\mathrm{SH}$ groups from the $\mathrm{CDs}$ in S3. The treated sample yields blue shifts greater than $30 \mathrm{~nm}$ in the PL emission when compared with S3 (Figure S3). 

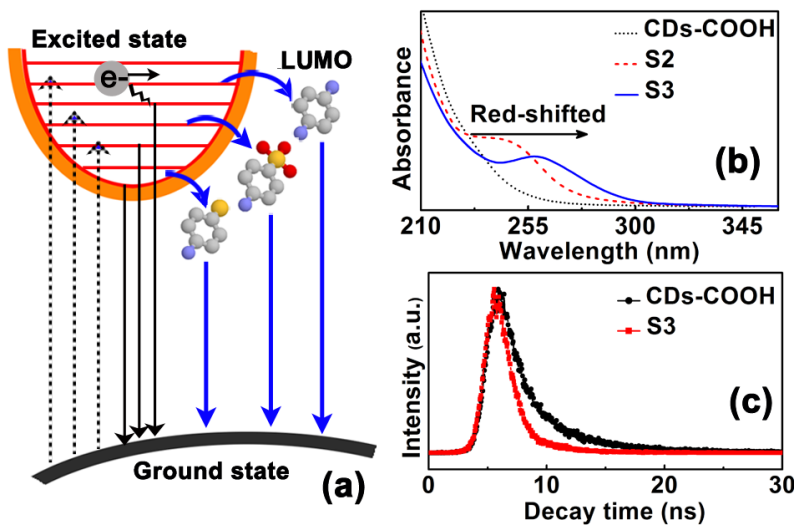

Figure 5. (a) Illustration for the PL mechanism of modified CDs; (b) Light absorption comparisons of CDs-COOH, S2, and S3; (c) PL decay profiles of CDs-COOH and S3 excited by the same wavelength.

As mentioned above, the amino-carboxylic molecular junction is an ultrafast electron transfer pathway [43], and consequently, the migration of excitation energy from the CDs to their superficial molecules is predominant due to the absence of long-time trips [36]. As shown in Figure 5c, PL decay studies reveal a markedly shortened lifetime of S3 (2.32 ns) in contrast with that of CD-COOH (5.40 ns), confirming the suppression of energy loss to the hole trapping and structural defects. Recently, some works also reported S-doped CDs, in which a variety of S-contained groups coexisted [25-28]. However, the PL emission of these S-doped CDs mainly exhibited blue or cyan colors and did not rely on the types of S-contained groups. To clarify whether the PL shifts between S2 and S3 are related to $\mathrm{S}$ contents in CDs or not, control experiments were conducted. During the surface modification for $\mathrm{CDs}-\mathrm{COOH}$, not only the reaction temperature but also the amount of added molecules such as sulfanilic acid and 4-aminothiophenol were changed. Although these factors could lead to large changes of the PL intensity, but they did not cause obvious shifts in the peak PL emission (Figures S4 and S5). Thereby, the charge transfers rather than the inter-band crossings are dominant in radiative recombination for molecule-modified CDs.

\section{Conclusions}

Molecules containing a benzene ring and different heteroatom substituents were employed to modify CDs through the acylation reaction, forming amino-carboxylic bonds. The different PL behaviors and emission colors were presented with the variations of heteroatom substituents in the used molecules. This could be attributed to photo-excited electron transfer from CDs to different LUMO levels of modified molecules via amino-carboxylic molecular junctions. Not only does this work provide a novel approach for tuning the emissive states in CDs and could be beneficial in understanding the rules governing the charge transfer from CDs to their surfaces, but it also enables new opportunities in the use of $\mathrm{CDs}$, since different heteroatom groups could lead to many probabilities for conjugation with ions and molecules. For instance, the CDs from S2 can be used for the selective detection of $\mathrm{Fe}^{3+}$ in live cells and aqueous solutions (Figure S6) owing to the introduction of $\mathrm{SO}_{3} \mathrm{H}$ groups. Our presented CDs are also expected to expand the choices for drug delivery, tracers, biosensing, and so on through the selective combination of heteroatom groups in CDs with drugs or organic molecules.

Supplementary Materials: The following are available online at www.mdpi.com/1996-1944/10/2/165/s1, Figures S1-S6.

Acknowledgments: This work was supported by the National Natural Science Foundation of China (Nos. 51272301, 51172214, U1510125, 51502270), the Shanxi Province Science Foundation for Youths (2014021008), the Specialized Research Fund for Sanjin Scholars Program of Shanxi Province, and the North University of China Fund for Distinguished Young Scholars. 
Author Contributions: S. Hu and Q. Chang conceived the experiments and wrote this paper; Y. Wang and W. Zhang performed the experiments and analyzed the data; J. Yang contributed analysis tools.

Conflicts of Interest: The authors declare no conflict of interest.

\section{References}

1. Li, H.; Kang, Z.; Liu, Y.; Lee, S.-T. Carbon nanodots: Synthesis, properties and applications. J. Mater. Chem. 2012, 22, 24230-24253. [CrossRef]

2. Ding, C.; Zhu, A.; Tian, Y. Functional surface engineering of C-Dots for fluorescent biosensing and in vivo bioimaging. Acc. Chem. Res. 2014, 47, 20-30. [CrossRef] [PubMed]

3. Himaja, A.L.; Karthik, P.S.; Singh, S.P. Carbon dots: The newest member of the carbon nanomaterials family. Chem. Rec. 2015, 15, 595-615. [CrossRef] [PubMed]

4. Lim, S.Y.; Shen, W.; Gao, Z. Carbon quantum dots and their applications. Chem. Soc. Rev. 2015, 44, 362-381. [CrossRef] [PubMed]

5. Liu, J.; Liu, Y.; Liu, N.; Han, Y.; Zhang, X.; Huang, H.; Lifshitz, Y.; Lee, S.-T.; Zhong, J.; Kang, Z. Metal-free efficient photocatalyst for stable visible water splitting via a two-electron pathway. Science 2015, 347, 970-974. [CrossRef] [PubMed]

6. Zheng, X.T.; Ananthanarayanan, A.; Luo, K.Q.; Chen, P. Glowing graphene quantum dots and carbon dots: Properties, syntheses, and biological applications. Small 2015, 11, 1620-1636. [CrossRef] [PubMed]

7. Güçlü, A.D.; Potasz, P.; Korkusinski, M.; Hawrylak, P. Single-Particle Properties of Graphene Quantum Dots. In Graphene Quantum Dots; Springer: Berlin/Heidelberg, Germany, 2014; pp. 39-90.

8. Güçlü, A.D.; Potasz, P.; Korkusinski, M.; Hawrylak, P. Electron-Electron Interactions in Graphene Quantum Dots. In Graphene Quantum Dots; Springer: Berlin/Heidelberg, Germany, 2014; pp. 91-110.

9. Ozfidan, I.; Güçlü, A.D.; Korkusinski, M.; Hawrylak, P. Theory of optical properties of graphene quantum dots. Phys. Status Solidi 2016, 10, 102-110. [CrossRef]

10. Ozfidan, I.; Korkusinski, M.; Hawrylak, P. Electronic properties and electron-electron interactions in graphene quantum dots. Phys. Status Solidi 2016, 10, 13-23. [CrossRef]

11. Li, X.; Zhang, S.; Kulinich, S.A.; Liu, Y.; Zeng, H. Engineering surface states of carbon dots to achieve controllable luminescence for solid-luminescent composites and sensitive Be ${ }^{2+}$ detection. Sci. Rep. 2014, 4, 4976. [CrossRef]

12. Bao, L.; Liu, C.; Zhang, Z.-L.; Pang, D.-W. Photoluminescence-tunable carbon nanodots: Surface-state energy-gap tuning. Adv. Mater. 2015, 27, 1663-1667. [CrossRef] [PubMed]

13. Hu, S. Tuning optical properties and photocatalytic activities of carbon-based "quantum dots" through their surface groups. Chem. Rec. 2016, 16, 219-230. [CrossRef] [PubMed]

14. Zhu, S.; Zhang, J.; Tang, S.; Qiao, C.; Wang, L.; Wang, H.; Liu, X.; Li, B.; Li, Y.; Yu, W.; et al. Surface chemistry routes to modulate the photoluminescence of graphene quantum dots: From fluorescence mechanism to up-conversion bioimaging applications. Adv. Funct. Mater. 2012, 22, 4732-4740. [CrossRef]

15. Strauss, V.; Margraf, J.T.; Dolle, C.; Butz, B.; Nacken, T.J.; Walter, J.; Bauer, W.; Peukert, W.; Spiecker, E.; Clark, T.; et al. Carbon nanodots: Toward a comprehensive understanding of their photoluminescence. J. Am. Chem. Soc. 2014, 136, 17308-17316. [CrossRef] [PubMed]

16. Wang, C.; Wu, X.; Li, X.; Wang, W.; Wang, L.; Gu, M.; Li, Q. Upconversion fluorescent carbon nanodots enriched with nitrogen for light harvesting. J. Mater. Chem. 2012, 22, 15522-15525. [CrossRef]

17. Wang, S.; Cole, I.S.; Zhao, D.; Li, Q. The dual roles of functional groups in the photoluminescence of graphene quantum dots. Nanoscale 2016, 8, 7449-7458. [CrossRef] [PubMed]

18. Tetsuka, H.; Asahi, R.; Nagoya, A.; Okamoto, K.; Tajima, I.; Ohta, R.; Okamoto, A. Optically tunable amino-functionalized graphene quantum dots. Adv. Mater. 2012, 24, 5333-5338. [CrossRef] [PubMed]

19. Nie, H.; Li, M.; Li, Q.; Liang, S.; Tan, Y.; Sheng, L.; Shi, W.; Zhang, S.X.-A. Carbon dots with continuously tunable full-color emission and their application in ratiometric $\mathrm{pH}$ sensing. Chem. Mater. 2014, 26, 3104-3112. [CrossRef]

20. Hu, S.; Trinchi, A.; Atkin, P.; Cole, I. Tunable photoluminescence across the entire visible spectrum from carbon dots excited by white light. Angew. Chem. Int. Ed. 2015, 54, 2970-2974. [CrossRef] [PubMed] 
21. Jiang, K.; Sun, S.; Zhang, L.; Lu, Y.; Wu, A.; Cai, C.; Lin, H. Red, green, and blue luminescence by carbon dots: Full-color emission tuning and multicolor cellular imaging. Angew. Chem. Int. Ed. 2015, 54, 5360-5363. [CrossRef] [PubMed]

22. Pan, L.; Sun, S.; Zhang, A.; Jiang, K.; Zhang, L.; Dong, C.; Huang, Q.; Wu, A.; Lin, H. Truly fluorescent excitation-dependent carbon dots and their applications in multicolor cellular imaging and multidimensional sensing. Adv. Mater. 2015, 27, 7782-7787. [CrossRef] [PubMed]

23. Ding, H.; Yu, S.-B.; Wei, J.-S.; Xiong, H.-M. Full-Color Light-Emitting Carbon Dots with a Surface-StateControlled Luminescence Mechanism. ACS Nano 2016, 10, 484-491. [CrossRef] [PubMed]

24. Guo, L.; Ge, J.; Liu, W.; Niu, G.; Jia, Q.; Wang, H.; Wang, P. Tunable multicolor carbon dots prepared from well-defined polythiophene derivatives and their emission mechanism. Nanoscale 2016, 8, 729-734. [CrossRef] [PubMed]

25. Dong, Y.; Pang, H.; Yang, H.B.; Guo, C.; Shao, J.; Chi, Y.; Li, C.M.; Yu, T. Carbon-based dots co-doped with nitrogen and sulfur for high quantum yield and excitation-independent emission. Angew. Chem. Int. Ed. 2013, 52, 7800-7804. [CrossRef] [PubMed]

26. Kwon, W.; Lim, J.; Lee, J.; Park, T.; Rhee, S.-W. Sulfur-incorporated carbon quantum dots with a strong long-wavelength absorption band. J. Mater. Chem. C 2013, 1, 2002-2008. [CrossRef]

27. Li, X.; Lau, S.P.; Tang, L.; Ji, R.; Yang, P. Sulphur doping: A facile approach to tune the electronic structure and optical properties of graphene quantum dots. Nanoscale 2014, 6, 5323-5328. [CrossRef] [PubMed]

28. Song, Z.; Quan, F.; Xu, Y.; Liu, M.; Cui, L.; Liu, J. Multifunctional N,S co-doped carbon quantum dots with $\mathrm{pH}$ - and thermo-dependent switchable fluorescent properties and highly selective detection of glutathione. Carbon 2016, 104, 169-178. [CrossRef]

29. Sun, X.; Bruckner, C.; Lei, Y. One-pot and ultrafast synthesis of nitrogen and phosphorus co-doped carbon dots possessing bright dual wavelength fluorescence emission. Nanoscale 2015, 7, 17278-17282. [CrossRef] [PubMed]

30. Xu, Q.; Pu, P.; Zhao, J.; Dong, C.; Gao, C.; Chen, Y.; Chen, J.; Liu, Y.; Zhou, H. Preparation of highly photoluminescent sulfur-doped carbon dots for Fe(III) detection. J. Mater. Chem. A 2015, 3, 542-546. [CrossRef]

31. Xu, Y.; Wu, M.; Liu, Y.; Feng, X.-Z.; Yin, X.-B.; He, X.-W.; Zhang, Y.-K. Nitrogen-doped carbon dots: A facile and general preparation method, photoluminescence investigation, and imaging applications. Chem. Eur. J. 2013, 19, 2276-2283. [CrossRef] [PubMed]

32. Kumar, G.S.; Roy, R.; Sen, D.; Ghorai, U.K.; Thapa, R.; Mazumder, N.; Saha, S.; Chattopadhyay, K.K. Amino-functionalized graphene quantum dots: Origin of tunable heterogeneous photoluminescence. Nanoscale 2014, 6, 3384-3391. [CrossRef] [PubMed]

33. Zhu, S.; Zhang, J.; Liu, X.; Li, B.; Wang, X.; Tang, S.; Meng, Q.; Li, Y.; Shi, C.; Hu, R.; Yang, B. Graphene quantum dots with controllable surface oxidation, tunable fluorescence and up-conversion emission. RSC Adv. 2012, 2, 2717-2720. [CrossRef]

34. Tang, L.; Ji, R.; Li, X.; Bai, G.; Liu, C.P.; Hao, J.; Lin, J.; Jiang, H.; Teng, K.S.; Yang, Z.; et al. Deep ultraviolet to near-infrared emission and photoresponse in layered n-doped graphene quantum dots. ACS Nano 2014, 8, 6312-6320. [CrossRef] [PubMed]

35. Jin, S.H.; Kim, D.H.; Jun, G.H.; Hong, S.H.; Jeon, S. Tuning the photoluminescence of graphene quantum dots through the charge transfer effect of functional groups. ACS Nano 2012, 7, 1239-1245. [CrossRef] [PubMed]

36. Khan, S.; Gupta, A.; Verma, N.C.; Nandi, C.K. Time-resolved emission reveals ensemble of emissive states as the origin of multicolor fluorescence in carbon dots. Nano Lett. 2015, 15, 8300-8305. [CrossRef] [PubMed]

37. Wang, X.; Cao, L.; Lu, F.; Meziani, M.J.; Li, H.; Qi, G.; Zhou, B.; Harruff, B.A.; Kermarrec, F.; Sun, Y.-P. Photoinduced electron transfers with carbon dots. Chem. Commun. 2009. [CrossRef] [PubMed]

38. Tian, R.; Hu, S.; Wu, L.; Chang, Q.; Yang, J.; Liu, J. Tailoring surface groups of carbon quantum dots to improve photoluminescence behaviors. Appl. Surf. Sci. 2014, 301, 156-160. [CrossRef]

39. Mu, Y.; Wang, N.; Sun, Z.; Wang, J.; Li, J.; Yu, J. Carbogenic nanodots derived from organo-templated zeolites with modulated full-color luminescence. Chem. Sci. 2016, 7, 3564-3568. [CrossRef]

40. Hu, S.; Tian, R.; Wu, L.; Zhao, Q.; Yang, J.; Liu, J.; Cao, S. Chemical Regulation of Carbon Quantum Dots from Synthesis to Photocatalytic Activity. Chem. Asian J. 2013, 8, 1035-1041. [CrossRef] [PubMed]

41. Hu, S.; Zhang, W.; Chang, Q.; Yang, J.; Lin, K. A chemical method for identifying the photocatalytic active sites on carbon dots. Carbon 2016, 103, 391-393. [CrossRef] 
42. Li, Q.; Ohulchanskyy, T.Y.; Liu, R.; Koynov, K.; Wu, D.; Best, A.; Kumar, R.; Bonoiu, A.; Prasad, P.N. Photoluminescent carbon dots as biocompatible nanoprobes for targeting cancer cells in vitro. J. Phys. Chem. C 2010, 114, 12062-12068. [CrossRef]

43. Kladnik, G.; Puppin, M.; Coreno, M.; de Simone, M.; Floreano, L.; Verdini, A.; Morgante, A.; Cvetko, D.; Cossaro, A. Ultrafast charge transfer pathways through a prototype amino-carboxylic molecular Junction. Nano Lett. 2016, 16, 1955-1959. [CrossRef] [PubMed] 